\title{
Thure von Uexkülls semiotisches Modell des Menschen als Grundlage für eine moderne Theorie der Homöopathie*
}

\author{
Josef M. Schmidt \\ Institut für Ethik, Geschichte und Theorie der Medizin, Ludwig-Maximilians-Universität München, Deutschland
}

\section{Schlüsselwörter}

Medizingeschichte $\cdot$ Medizintheorie $\cdot$ Homöopathie .

Samuel Hahnemann · Thure von Uexküll · Jakob von Uexküll

\section{Zusammenfassung}

Während die konventionelle moderne Medizin ihr Selbstverständnis und ihre Praxis weitgehend am Vorbild der konventionellen Naturwissenschaft orientiert, erhebt die Homöopathie den Anspruch, Patienten nicht reduktionistisch, sondern individuell und ganzheitlich zu erfassen und zu behandeln. Gemäss der Lehre Samuel Hahnemanns (1755-1843) ist dazu statt des materialistisch-mechanistischen ein semiotisch-phänomenologischer Ansatz anzuwenden. Während die Anweisungen Hahnemanns für die Praxis klar, deutlich und brauchbar sind, gibt es immer noch keine schlüssige und allgemeingültige Theorie der Homöopathie. Die methodologischen Eigenheiten der Homöopathie lassen sich allerdings mithilfe des semiotischen Modells des Menschen konzeptualisieren und erklären, das Thure von Uexküll (1908-2004) für die Psychosomatik ausgearbeitet hat und das auf der Lehre Jakob von Uexkülls (1864-1944) von den Funktionskreisen und spezifischen Umwelten beruht. Wird der Patient als biopsychosoziale Einheit mit seiner individuellen Wirklichkeit begriffen, lassen sich Begriffe und Konzepte wie die Verstimmung der Lebenskraft oder Idiosynkrasien, aber auch Arzneimittelwirkungen und die Arzt-PatientBeziehung auf eine moderne, wissenschaftlich begründete und anschlussfähige Weise verstehen.

\footnotetext{
*Überarbeitete Version eines Vortrags, gehalten anlässlich des 6. Köthener Sommerkurses Homöopathiegeschichte am 03.09.2011 in der Europäischen Bibliothek für Homöopathie in Köthen (Anhalt) Deutschland.
}

\author{
Keywords \\ History of medicine - Theory of medicine - Homeopathy . \\ Samuel Hahnemann · Thure von Uexküll · Jakob von Uexküll
}

\section{Summary \\ Thure von Uexküll's Semiotic Model of Man as a Basis for a New Theory of Homeopathy}

While conventional modern medicine's self-conception and practice is mainly oriented toward the paradigm of conventional science, homeopathy claims to perceive and treat patients not in a reductionistic but individual and holistic manner. According to the doctrine of Samuel Hahnemann (1755-1843), a semiotic and phenomenological instead of a materialistic and mechanistic approach has to be taken. While the directions for practice given by Hahnemann are clear, distinct, and feasible, a consistent and generally accepted theory of homeopathy is still lacking. The methodological peculiarities of homeopathy, however, can be conceptualized and explained by means of the semiotic model of man which Thure von Uexküll (1908-2004) had elaborated for psychosomatics on the basis of Jakob von Uexküll's (1864-1944) doctrine of functional cycles and specific environments. If the patient is conceived as a biopsychosocial unit with his/her individual reality, Hahnemann's notions and conceptions, such as derangement of the life force or idiosyncrasies as well as drug action and the physicianpatient relationship, may be comprehended in a modern, scientifically sound and connectable way.

\section{KARGER \\ Fax +497614520714 Information@Karger.d} www.karger.com
PD Dr. med. Dr. phil. Josef M. Schmidt

Institut für Ethik, Geschichte und Theorie der Medizin

Ludwig-Maximilians-Universität München

Lessingstrasse 2, 80336 München, Deutschland

j.m.schmidt@lrz.uni-muenchen.de 


\section{Mots-clés}

Histoire de la médecine · Théorie de la médecine · Homéopathie

Samuel Hahnemann · Thure von Uexküll · Jakob von Uexküll

\section{Résumé}

Modèle sémiotique de l'homme par Thure von Uexküll comme base d'une théorie moderne de l'homéopathie

Alors que la médecine conventionnelle moderne continue de s'orienter en théorie comme en pratique, vers le modèle des sciences naturelles conventionnelles, I'homéopathie affirme qu'il faut comprendre et traiter les patients non pas de manière réductrice, mais de manière individuelle et globale. Selon la théorie de Samuel Hahnemann (17551843), il convient d'adopter une approche sémiotique et phénoménologique plutôt que matérialiste et mécaniste. Alors que les consignes de Hahnemann pour la pratique sont claires, intelligibles et applicables, il n'existe cependant pas de théorie de l'homéopathie concluante et générale. Les propriétés méthodologiques de l'homéopathie peuvent toutefois se conceptualiser et s'expliquer grâce au modèle sémiotique de l'homme élaboré par Thure von Uexküll (1908-2004) pour la psychosomatique, qui repose sur la notion d'Umwelt (environnement spécifique) et des cercles fonctionnels de Jakob von Uexküll (18641944). Si I'on considère le patient comme une unité biopsychosociale dans sa propre réalité, on peut compendre de manière moderne, scientifique et cohérente les concepts tels que l'idiosyncrasie, les effets des médicaments ou le rapport médecin-patient.

\section{Nutzen und Grenzen der Wissenschaft}

Dass die moderne Wissenschaft ein mächtiges Werkzeug zur Lösung von Problemen auf vielen Gebieten unseres Lebens ist, wird heute niemand ernsthaft bezweifeln. Jeder profitiert von den Erleichterungen, die wissenschaftlicher Fortschritt den Menschen brachte - vom elektrischen Licht bis zu modernen Verkehrs- und Kommunikationsmitteln. Beeindruckend sind auch die vielen prestigeträchtigen Entdeckungen etwa der Kosmologie und Atomphysik, ermöglicht durch Raumfahrt, Weltraumteleskope oder Teilchenbeschleuniger. Bei näherer Betrachtung erweisen sich jedoch viele Errungenschaften und Anwendungen moderner Wissenschaft nicht nur als nützlich für die Menschheit, sondern als risikoträchtig und gefährlich, wie es am Beispiel der Kernenergie offenkundig ist. Je schädlicher sich wissenschaftliche Technologien auf Lebewesen auswirken, desto stärker wird dann aber das Bedürfnis nach einer medizinischen Wissenschaft, um die Kollateralschäden und unerwünschten Nebenwirkungen wissenschaftlichen Fortschritts auf die Gesundheit der Menschen zu reparieren.

Die konventionelle moderne Medizin stützt sich allerdings auf dieselben Prinzipien und Methoden wie die konventionellen Naturwissenschaften. In Gebieten wie der Epidemiologie, Bakteriologie, Toxikologie usw. erweist sie sich damit als äusserst kompetent und hilfreich. Stark und überzeugend ist sie im Allgemeinen dort, wo es möglich ist, sich medizinischen Problemen auf dem Weg der Verallgemeinerung, Quantifizierung und der Statistik zu nähern. Wie Physiker und Chemiker beschränken sich auch konventionelle medizinische Wissenschaftler methodisch auf die Suche nach allgemeingültigen Naturgesetzen, in Form von Kausalität, Mechanismus, Ökonomie und Effektivität.

Sie versuchen daher, Krankheiten, Medikamentenwirkungen und Korrelationen zwischen den Körperteilen so zu erforschen, als ob dies neutrale Objekte oder Entitäten wären, die unabhängig von einem besonderen Kontext existierten. Verführt durch spektakuläre Erfolge bei der Kontrolle und Beherrschung von lebensbedrohlichen $\mathrm{Zu}-$ ständen des Organismus und bei der Messung und Manipulation immer kleinerer Strukturen und Funktionen der Zellen, Gene und Moleküle, befinden sich heute in der Tat die meisten Wissenschaftler, Wissenschaftsjournalisten und Laien in Versuchung, zu meinen, dass wahrscheinlich alles in der Medizin auf diese Weise erforscht werden könne - wenn nicht sogleich, so doch eines Tages in der Zukunft.

\section{Konventionelle Medizin und Wissenschaft}

Diese weitverbreitete Haltung eines verallgemeinerten Wissenschaftspositivismus kann jedoch - vor allem im Bereich der Medizin - auf vielfältige Weise hinterfragt werden und erweist sich in der Tat als unhaltbar, wenn man sie systematisch untersucht $[1,2]$.

Versucht man, die kritischen Argumente und Einwände auf eine kurze Formel zu bringen, könnte man sagen, dass die moderne Wissenschaft an einer Anfangsvergessenheit leidet, an einem Nicht-Gewahrsein ihres blinden Flecks oder an der Illusion ihrer Autonomie. Das Problem ist, dass Wissenschaftler dazu neigen, zu vergessen, dass das, was sie tun, viel mehr ist, als nur Messwerte zu registrieren und zu sammeln. Dies können auch Roboter und Computer. Eine Wissenschaft zu betreiben ist jedoch eine menschliche Tätigkeit, die menschliche Subjekte voraussetzt, die wiederum nie auf passive Wahrnehmung oder Anpassung an vermeintlich objektive äussere Bedingungen beschränkt sind, sondern immer auch die Welt um sich herum konstruieren und interpretieren. Das Betreiben einer Wissenschaft ist daher kein harmloser, vermeintlich neutraler Brotberuf, sondern hat unvermeidbar höchste praktische und ethische Implikationen.

Philosophisch gesehen, beruht menschliche Erkenntnis immer auf bestimmten, dem Erkenntnisakt jeweils zu- 
grunde liegenden Begriffen, Konzepten und Paradigmen, die vom menschlichen Denken ins Spiel gebracht werden. Gleichzeitig aber ist menschliche Erkenntnis immer auch in Gefahr, durch eben diese zugrunde gelegten Begriffe, Konzepte und Paradigmen fehlgeleitet, verzerrt oder verfälscht zu werden [3].

Wissenschaft, besonders die moderne Wissenschaft, die sich vor ungefähr 300 Jahren konstituierte und die seit ca. 150 Jahren die konventionelle Medizin dominiert, tendiert traditionellerweise dazu, sich auf einen Kanon methodischer Regeln und Gesetze zu fixieren. Statt aber ständig deren Geltungsbereich und deren Grenzen zu berücksichtigen, versucht die moderne konventionelle Medizin, so viel wie möglich mit dieser Methodik zu erklären - mittels Reduktion, Subsumtion und Generalisierung.

Philosophie, Poesie und Kunst - wie auch Wissenschaftsgeschichte und Wissenschaftstheorie - sind demgegenüber kontinuierliche Versuche, die Dominanz und Ausschliesslichkeit der gerade vorherrschenden Paradigmen und Geisteshaltungen zu hinterfragen, herauszufordern und zu widerlegen. Sie versuchen vielmehr, neue Räume zu eröffnen, neue Kategorien zu finden oder neue Freiheiten zu beanspruchen, um dadurch das Erscheinen von Phänomenen $\mathrm{zu}$ ermöglichen, die sonst gar nicht am Horizont der Wahrnehmung auftauchen und sichtbar werden könnten.

Der Befund einer Anfangsvergessenheit der modernen Wissenschaft ist aber nicht nur ein theoretisches Problem, sondern erweist sich auch praktisch als hochrelevant. Dieser strukturelle Mangel ist der Grund zahlreicher Probleme, mit denen die Medizin heute konfrontiert ist. Moderne Wissenschaftler, die z.B. versuchen, das Gedächtnis des Körpers oder der Seele zu erforschen, sind durch die wissenschaftliche Methode gebunden, nach vermeintlich objektiven Entitäten zu suchen, wie molekularbiologischen Engrammen, chemischen Überträgersubstanzen, Nervenströmen, Gehirnstrukturen usw. Sie nehmen einen distanzierten Standpunkt ein und betrachten stets nur den Körper eines anderen.

Es ist dies jedoch ein exoterischer Blick von aussen und die reservierte Haltung eines vermeintlich neutralen Beobachters auf vermeintlich von ihm getrennte, entfernte Objekte [4]. Die innere Dimension dessen, was wir Gedächtnis nennen, seine Funktion, seine Bedeutung und seine Dynamik können aber nicht bereits dadurch als verstanden gelten, dass man seine notwendigen physikalischen und chemischen Bedingungen aufzählt. An dieser Stelle fordert die Kategorie der Subjektivität, die lange in der wissenschaftlichen Medizin vernachlässigt wurde, ihren legitimen konstitutiven Platz in der Medizintheorie.

\section{Der Patient als «Subjekt» in der Medizin}

Im 20. Jahrhundert wurden bedeutende Versuche unternommen, das Subjekt in die Biologie und Medizin einzuführen, etwa durch den Physiologen und Medizinphilosophen Viktor von Weizsäcker (1886-1957) [5]. Bislang ist allerdings nicht erkennbar, dass dieses Projekt zu einer nennenswerten Verunsicherung des konventionellen Modells der Mainstream-Medizin geführt hätte [6]. Die Einführung des Subjekts in die Medizin war aber auch nicht als blosse Addition eines zusätzlichen Begriffs zu einer Sammlung von mechanischen Werkzeugen und Konzepten gemeint, sondern hätte einen paradigmatischen Wechsel bedeutet, quasi eine Dekonstruktion bzw. Rekonstruktion des fundamentalen wissenschaftlichen Rahmens der Medizin.

Seit der Definition von Tieren als Automaten von René Descartes (1596-1650) und der Vergegenständlichung von Menschen als Maschinen [7] durch Julien Offray de la Mettrie (1709-1751) verwenden Physiker und Chemiker, aber zunehmend auch die sogenannten Lebenswissenschaften (Life Sciences im weiteren Sinne, einschliesslich Biologie, Medizin, Psychologie usw.) dieselben reduktionistischen Kriterien wissenschaftlicher Forschung. Naturwissenschaftler haben es allerdings mit toten Objekten zu tun, wie Massen, Kräften, Drücke usw., während Lebenswissenschaftler doch für die Phänomene des Lebendigen zuständig sind. Grundsätzlich versuchte jede Branche der Wissenschaft, die ganze Welt, die belebte wie die unbelebte, auf mechanische, physikalische, chemische, mathematische oder statistische Gesetze und kausale Verknüpfungen zu reduzieren. Dies hatte und hat einen mächtigen Einfluss auf die moderne Medizin, unser Menschenbild sowie die Homöopathie.

Das heute verbreitete Menschenbild wird von der Art und Weise bestimmt, wie die konventionelle moderne Medizin dessen Teile und Funktionen untersucht. Dementsprechend werden Menschen in der Regel als komplizierte Mechanismen aufgefasst, Gesundheit als deren regelmässige und effiziente Funktion und Krankheit als deren Versagen, was durch Messwerte objektivierbar sein soll. Als Nebeneffekt der wissenschaftlichen Methode werden dann Medikamentenwirkungen als kausale Einwirkungen auf den Körper aufgefasst, wie bei einer chemischen Reaktion, physikalischen Unterdrückung oder materiellen Substitution. Überspitzt ausgedrückt, wird im konventionellen wissenschaftlichen Ansatz kein Unterschied gemacht zwischen der Kausalität der Arzneiwirkung in vitro und in vivo. Der Substanz wird unterstellt, dass sie den ihr zugeschriebenen Effekt ausübt - mit oder ohne Subjekt des Patienten.

Werden diese Koordinaten und Variablen zum Ausgangspunkt einer Bewertung jeder Art von Medizin ge- 
nommen, so ist klar, wie vor diesem Hintergrund z.B. die Homöopathie erscheint. Aus Sicht der Evidence-based Medicine, dem alles beherrschenden Instrument, um die modernen wissenschaftlichen Standards in der Medizin im grossen Stil einzuführen, erfüllt die Homöopathie in der Regel nicht die Kriterien biomedizinischer Technologien, die sämtlich standardisierbar, quantifizierbar und statistisch determinierbar zu sein haben. Bei einer naiven Schlussfolgerung klingt dieses Verdikt vonseiten führender medizinischer Autoritäten für eine breite Öffentlichkeit dann so, als ob damit die Unwissenschaftlichkeit der Homöopathie erwiesen wäre [8].

Anders interpretiert, zeigt dieses Paradox womöglich aber nur die Inkompatibilität von konventioneller medizinischer Methodik einerseits und Praxis und Kunst der Homöopathie andererseits. Das Ergebnis liesse sich demnach eher als ein Versagen des konventionellen biomedizinischen Paradigmas deuten denn als Widerlegung der homöopathischen Heilmethode. Anstatt in negativen Studienergebnissen immer gleich einen Mangel an Evidenz zu sehen, wäre hier zu überlegen, ob nicht vielmehr Evidenz für einen Mangel vorliegt, etwa einen Mangel an Validität, Verständnis und Durchdachtheit der Methode.

Die Herausforderung für Homöopathen besteht demnach eher darin, eine eigene wissenschaftliche Theorie $\mathrm{zu}$ finden und zu etablieren, als sich an externe Standards der konventionellen Medizin anzupassen und zu versuchen, theoretisch damit zurechtzukommen. Darüber hinaus besteht die Herausforderung für die Medizin als Ganze, also orthodoxer, heterodoxer und alternativer Richtungen zusammen, darin, eine Theorie der Medizin zu entwickeln, die weit genug ist, um zu erklären, was sowohl konventionelle als auch z.B. homöopathische Ärzte treiben. Immerhin behandeln beide Schulen Lebewesen. Insofern muss jede Theorie, die nicht auch spezifische Phänomene des Lebendigen impliziert, wie Subjektivität, Beziehungen und Kommunikation, hinter dem wirklichen Leben zurückbleiben.

\section{Uexkülls semiotisches Modell des Menschen}

Auf der Suche nach einer Theorie der Medizin, die umfassend genug ist, um auch einen konzeptuellen Rahmen für die Homöopathie zu bieten, könnte sich das Werk des deutschen Arztes baltischer Herkunft Thure von Uexküll (1908-2004) als ein wertvoller Schlüssel erweisen. Uexküll war im 20. Jahrhundert einer der Protagonisten des Versuchs, das Subjekt in die medizinische Theorie einzuführen [9]. Seit den 1950er Jahren profilierte er sich als einer der Begründer der psychosomatischen Medizin in Deutschland, indem er das sogenannte biopsychosoziale Modell des Menschen, wie es von George Engel (1913-
1999) vorgeschlagen wurde [10], ausarbeitete. Seine Grundidee und Vision war, ein wissenschaftliches Modell des Menschen zu etablieren, das einerseits den Reduktionismus, Mechanismus und Materialismus der konventionellen wissenschaftlichen Methode überwindet und andererseits dem Arzt ermöglicht, den Patienten als Einheit in vielerlei Hinsicht zu verstehen, etwa als Einheit von verschiedenen Ebenen der Existenz, wie vegetative, animalische und mentale Funktionen, aber auch als Einheit zwischen Organismus und Umwelt, kurz zwischen Subjekt und Objekt [11].

In der konventionellen Wissenschaft berücksichtigen weder Physiologen, Psychologen noch Soziologen den ganzen Menschen, sondern stattdessen wendet jeder die Methode, Konzepte und Paradigmen seines spezifischen Fachgebietes an, beobachtet dadurch unterschiedliche Dinge und spricht eine andere Sprache als die seiner externen Kollegen.

Im Gegensatz dazu versuchte Uexküll, seine Theorie auf Kategorien zu begründen, die breit genug sind, um sie auf die verschiedenen Ebenen, Dimensionen und Beziehungen des Patienten anzuwenden. Dazu verwendete er kybernetische, semiotische (zeichentheoretische) und konstruktivistische Konzepte aus der Systemtheorie. Diese Ansätze waren in der Regelungstechnik, Linguistik, Philosophie und Soziologie entwickelt worden, aber noch nicht in die Medizin eingeführt.

Vorbereitende Arbeiten, auf die sich Thure von Uexküll stützen konnte, waren bereits von seinem Vater, Jakob von Uexküll (1864-1944), durchgeführt und publiziert worden, einem deutsch-baltischen Zoologen und Pionier der theoretischen Biologie. Er war in Estland geboren, ging in Reval (Tallin) zur Schule und graduierte 1890 an der Universität von Dorpat (Tártu). Inspiriert von seinen Studien Immanuel Kants (1724-1804) und deutscher und baltischer romantischer Naturforscher, wie Johannes Müller (1801-1858) und Karl Ernst von Baer (1792-1876), führte er zu Beginn des 20. Jahrhunderts das Konzept des «Funktionskreises» und das der «spezifischen Umwelt» von Tieren ein, womit er die traditionelle Unterstellung einer vermeintlichen Objektivität, die unabhängig von einzelnen Subjekten existieren sollte, herausforderte [12, 13]. Der herkömmlichen kantianischen Vorstellung einer gemeinsamen Welt von Objekten bzw. eines «Dinges an sich" stellte er das Konzept einer sinndeutenden Biologie gegenüber [14].

Lange bevor moderne Konstruktivisten und Neurowissenschaftler zu behaupten begannen, dass das, was wir Wirklichkeit nennen, eher ein Bild, eine Idee oder eine Illusion sei als eine objektive unveränderliche Welt, die eine und dieselbe für jeden wäre, kam Jakob von Uexküll zu ähnlichen Schlussfolgerungen durch seine Experimente mit Seeigeln und anderen Meeres- und Erdentieren. 
Aufgrund empirischer Forschung kam er zu dem Ergebnis, dass jedes Lebewesen seine subjektive Umwelt konstruiert und damit auch kontrolliert [15-17]. Wie sich z.B. die Umwelt einer Zecke (Ixodida) anfühlt bzw. wie sie aussieht, könne von deren sensorischen und motorischen Organen deduziert werden. Die Zecke könne nur die Sinnesqualitäten warm oder kalt, oben oder unten sowie Vorhandensein oder Abwesenheit von Buttersäure wahrnehmen und darauf reagieren. Dementsprechend habe nur solche Information eine Bedeutung für die Zecke, während alles andere für sie bedeutungslos und nicht wahrnehmbar sei und daher nie Teil ihrer subjektiven Umwelt werde [18].

Unterschiedliche Lebewesen erteilen den Phänomenen, die sie wahrnehmen, unterschiedliche Bedeutung. Aber auch dasselbe Individuum kann einer bestimmten Sache für eine begrenzte Zeit grosse Bedeutung erteilen, während es dieselbe zu einer anderen Zeit als bedeutungslos empfindet. Dieser Unterschied in der Bewertung hängt vom inneren Zustand des Lebewesens ab. Wenn es hungrig ist, erlangen potenzielle Nahrungsmittel die höchste Bedeutung innerhalb seiner wahrnehmbaren Welt. Wenn es einen Sexualpartner sucht, konzentriert sich seine Wahrnehmung auf andere Dinge und formt so eine andere subjektive Umwelt, solange eben diese Präferenz andauert. Der schwierige Punkt, den es hier zu verstehen gilt, ist, dass niemand von einer neutralen objektiven Realität wissen kann, in der alle Lebewesen gleichermassen leben und interagieren. Stattdessen lebt jeder in seiner subjektiven Umwelt, die das Ergebnis eines ständigen Konstruktionsprozesses ist, der wiederum vom jeweils vorherrschenden eigenen inneren Zustand abhängt.

Die fundamentale Lebenseinheit kann daher als ein Funktionskreis konzeptualisiert werden, dessen sensorischen und motorischen Arm ein zirkulärer semiotischer Prozess verbindet. Je nach seinen aktuellen Bedürfnissen nimmt der Organismus Zeichen aus seiner Umwelt auf, denen er eine Bedeutung erteilt und auf die er auf eine Weise reagiert, die diese Zeichen betrifft und verändert, sodass deren Bedeutung und dadurch seine Umwelt sich ändern. Im Gegensatz und komplementär zur konventionellen kausal-mechanischen Erklärung beruht dieser neue Ansatz, die Interaktionen zwischen Lebewesen und ihrer spezifischen Umwelt zu verstehen, auf dem Konzept der Subjektivität. Deshalb ist er nicht deterministisch, sondern hermeneutisch, weil eben die vielfältige Bedingtheit der Lebensphänomene der kontextuellen Auslegung und Interpretation bedarf.

Indem Thure von Uexküll den innovativen und richtungsweisenden Ansatz seines Vaters hinsichtlich eines wissenschaftlichen Verständnisses des Lebendigen fortführte, arbeitete er dessen Grundmodell eines Funktionskreises für die Bedingungen des Menschseins aus und ergänzte es durch sein Konzept des «Situationskreises».
Neben der zunehmenden Komplexität hinsichtlich zusätzlicher psychischer und sozialer Ebenen, die es beim Menschen zu berücksichtigen gilt, sah er ein Hauptspezifikum der conditio humana in der Möglichkeit des menschlichen Geistes, statt direkt auf wahrgenommene Zeichen zu reagieren, sich diese in einer Situation nur vorzustellen und sozusagen probezuhandeln, ohne ein Risiko einzugehen. Um diese spezifisch menschliche Möglichkeit in Begriffen der Systemtheorie zu beschreiben, prägte er das Konzept des Situationskreises.

Die wirkliche Stärke des biopsychosozialen Modells des Menschen liegt jedoch in der Fähigkeit, die theoretische wie auch institutionelle Trennung der physiologischen, psychischen und sozialen Probleme des Patienten zu überwinden [19]. Egal, welche Ebene der Existenz man betrachtet, ob biologische Zellfunktion, Interaktion von Organen, psychische Kommunikation oder soziale Beziehungen das semiotische (zeichentheoretische) Modell der Erteilung, Verarbeitung und Transformation der Bedeutung wahrgenommener Zeichen und damit der kontinuierlichen Rekonstruktion der eigenen subjektiven Umwelt erweist sich als anwendbar auf alle Fälle. Da jede Zelle, jedes Organ, jeder Organismus seine eigene Umgebung, seine eigene Wichtigkeitsordnung und seinen eigenen Code hat, um bedeutungsvolle Zeichen zu entdecken und $\mathrm{zu}$ verarbeiten, müssen die Interaktionen zwischen verschiedenen Systemen oder Ebenen als Übersetzungsprozesse (von einem Code in einen anderen) interpretiert werden und nicht als kausal-mechanische Wirkungen, die in der psychologischen und sozialen Dimension sowieso keine Erklärungskraft besitzen.

Die Konsequenz dieses Modells semiotischer und kybernetischer Prozesse, die einer permanenten Rekonstruktion unserer Umwelt zugrunde liegen, ist, dass jeder in seiner eigenen individuellen Wirklichkeit lebt. Die Konstruktion einer gemeinsamen Wirklichkeit ist zwar möglich (z.B. zwischen Arzt und Patient), bedarf aber einer besonderen Kommunikation und Zeichenübersetzung zwischen den Individuen und ihren subjektiven Umwelten [20]. Um die Dinge zu verkomplizieren, hat jede Einheit eines Subjekts und seiner Umwelt auch seine individuelle Geschichte, die an dem Punkt in der frühen Kindheit beginnt, an dem die fundamentale Einheit zum ersten Mal aufgebrochen ist, als beide, Subjekt und Objekt, entstanden, sich sozusagen aus der Nicht-Existenz erhoben und zur Welt gekommen sind.

Bei der Zurückverfolgung der Entwicklung des Selbst, seiner Sinne, seiner ersten Eindrücke, Gefühle und Konzepte bis zu dem Punkt, wo die im biopsychosozialen Modell beschriebenen systemischen Prozesse noch nicht vollzogen wurden, sondern erst geschaffen und initiiert werden mussten, gebraucht Thure von Uexküll das Wort «Stimmung», um diesen genuinen Zustand am Rande der 
wissenschaftlichen Sprache und Begriffe zu charakterisieren. Dieser Begriff lässt sich wegen seiner vielen Facetten nicht leicht in ein englisches Synonym übersetzen. Man müsste mehrere Wörter verwenden, um seinen Bedeutungsumfang wiederzugeben: etwa «mood», «atmosphere», «general feeling», «sentiment», «tone» oder «tuning».

Eine Art Stimmung wäre also der Vorläufer jeder kleinsten Einheit von Kommunikation, als minimale Form des Lebens. Durch die Sozialisation des Neugeborenen wird diese vorsprachliche Erfahrung später allmählich übersetzt und transformiert von einer vegetativen über eine animalische auf eine menschliche Ebene, wenn Strategien und Programme der Wahrnehmung und Tätigkeit zur Lösung von Problemen entstehen.

Andererseits ist aber auch im Erwachsenalter jeder biopsychosoziale Zustand oder Konflikt immer mitkonstituiert von einer Reproduktion und Realisation dieser originären individuellen Stimmung und deren Schicksal innerhalb der Biographie des Patienten [21].

\section{Die Verbindung zur Homöopathie}

Diese Wortwahl, die in der konventionellen «Hardcore»Wissenschaft sonst nicht üblich ist, erinnert den Medizinhistoriker an die bekannte Definition von Krankheit, die Samuel Hahnemann (1755-1843) vor etwa 200 Jahren bei dem Versuch, eine vorläufige Theorie seiner neuen Wissenschaft der Heilkunst darzulegen, eingeführt hat. Krankheit, sagte er, sei eine «Verstimmung» der Lebenskraft. Damit gebrauchte er die Wurzel desselben Begriffs («Stimmung»). Dementsprechend sei Heilung die Rückführung der Lebenskraft in den ursprünglichen Zustand der (guten) Stimmung [22].

Als Hahnemann versuchte, seinen Zeitgenossen in theoretischen Begriffen zu erklären, was er gefunden hatte und was er praktisch ausübte, war die Medizintheorie seiner Zeit gerade dabei, traditionelle Begriffe und Konzepte wie Semiologie, Teleologie und Vitalismus zu verlassen und sich modernen Ideen und Programmen wie Kausalität, Mechanismus und Materialismus zuzuwenden. Hahnemann, der an der Schnittstelle zweier ungleicher Epochen lebte und wirkte, bediente sich daher inhomogener Komponenten medizinischer Theorie - um eben anschlussfähig zu sein an den wissenschaftlichen Diskurs jener Übergangszeit. Dies ist der Grund, weshalb einige von Hahnemanns Konzepten heute antiquiert und veraltet, einige modern und progressiv und wieder andere postmodern oder sogar zeitlos gültig erscheinen.

Die Homöopathie als Ganze entbehrt jedoch immer noch einer konsistenten und schlüssigen Theorie. In der Tat, aufgrund des mangelnden Bewusstseins ihrer theoretischen Wurzeln tendieren Homöopathen bei Kritik vonseiten der «Wissenschaft» leicht zur Kapitulation und versuchen, sich mit den Anforderungen zu arrangieren, von denen sie denken, dass sie gegenwärtiger wissenschaftlicher Standard seien. Um zu verhindern, von der eigenen praktischen Erfahrung durch unangemessene theoretische Einwände entfremdet zu werden, ist jedoch ein gutes Verständnis der Stärken und Schwächen der jeweiligen Theorie vonnöten. Mit dem biopsychosozialen Modell des Menschen können jedenfalls die meisten Einwendungen vonseiten der konventionellen wissenschaftlichen Medizin entschärft oder aber integriert werden. Auf diese Weise könnte ein neues Selbstbewusstsein unter Homöopathen entstehen, wenn sie nämlich gewahr würden, dass das, was sie in der Praxis tun, sich in voller Übereinstimmung mit jenem Modell befindet, das als «das gegenwärtig kohärenteste, kompakteste und auch bedeutendste Theoriekonzept, innerhalb dessen der Mensch in Gesundheit und Krankheit erklärbar und verstehbar wird», angesehen wird [23].

Hält man dagegen am konzeptuellen Rahmen der konventionellen Medizin fest, ist es in Bezug auf die meisten traditionellen homöopathischen Begriffe und Konzepte nicht möglich, einen Sinn darin zu erkennen, etwa bei: Lebenskraft, Verstimmung, dynamische Wirkung, Zeichen, Idiosynkrasie usw. Diese können jedoch in die Sprache des semiotischen biopsychosozialen Modells übersetzt und innerhalb seiner Kategorien verstanden und verifiziert werden. Das Problem liegt möglicherweise eher auf der Seite der konventionellen medizinischen Theorie als auf der der homöopathischen Methodik und Praxis.

Um Missverständnissen und Fehlinterpretationen vorzubeugen, wären Homöopathen freilich gut beraten, konsequent Hahnemanns irreführende Begriffe zu vermeiden und sie durch theoretisch konsistente Ausdrücke zu ersetzen.

\section{Ansatzpunkte für eine moderne Theorie der Homöopathie}

Einige Beispiele mögen das Potenzial von Uexkülls semiotischem Modell des Menschen für eine moderne Theorie der Homöopathie illustrieren.

Begriffe und Konzepte wie Lebenskraft, Lebensprinzip oder Vitalkraft sind inkompatibel mit der methodologischen Reduktion der konventionellen Medizin auf Mechanismus, Materialismus und Positivismus. Im semiotischen Modell des Menschen als Subjekt gibt es dagegen kein Problem, Vitalität als konstitutive Dimension aller Phänomene des Lebendigen anzuerkennen. Im Gegenteil, seine ganze Theorie basiert darauf. Aus theoretischen Gründen ist es allerdings besser, von Intentionalität oder Subjektivität $\mathrm{zu}$ sprechen. Als autopoietisches (selbst- 
erschaffendes und -erhaltendes) Programm oder Regelkreis konzeptualisiert, ist dieser Begriff leichter anschlussfähig an andere Wissenschaften.

Individuelle Unterschiede wie Empfindsamkeit, Konstitution und Disposition von Patienten stellen ein fundamentales Problem für die konventionelle Medizin dar, deren methodologisches Instrumentarium vor allem auf Mittelwertvergleichen vor dem Hintergrund des Paradigmas von Ursache-Wirkungs-Zusammenhängen basiert. Die Tatsache, dass menschliche Individuen, je nach momentanem Zustand und persönlicher Geschichte, verschieden auf ein und dieselbe Substanz reagieren, findet dagegen ihre volle Anerkennung im semiotischen Modell mit den grundlegenden Zeichen-Prozessen der Funktionskreise. Entsprechend dem eigenen inneren Zustand (einschliesslich der Erinnerung an vergangene Zustände der individuellen Entwicklung) kann ein Patient zu einem Zeitpunkt einem bestimmten Arzneimittel eine hohe Bedeutung erteilen, während er zu einem anderen Zeitpunkt, in einem veränderten Zustand, einem anderen höchste Bedeutung erteilt. Ein Staphisagria-Zustand muss nicht ewig anhalten, sondern kann z.B. in einen PulsatillaZustand übergehen usw.

Bei derartigen Fällen würde sich die konventionelle Medizin entweder auf mögliche kausale Wirkungen auf der Ebene der Molekularbiologie konzentrieren oder aber sogleich zu psychosozialen Unterstellungen übergehen, wie Hypochondrie, Suggestion oder Placebo-Effekt. Im semiotischen Modell dagegen wird der Patient konsequent als Einheit angesehen und seine Reaktion auf ein Mittel wird auf eine einheitliche (zeichendynamisch rückgekoppelte) Weise verstanden, indem alle Ebenen seiner Existenz gleichzeitig abgedeckt werden. Im Gegensatz zum konventionellen Paradigma, demzufolge ohne materielle Substanz keine Arzneiwirkung möglich sei, ist im semiotischen Modell die Kontroverse um ultramolekulare Verdünnungen irrelevant, da der Ausgangspunkt hier weder eine vermeintlich objektive Welt noch der Begriff der Materie, sondern die Subjektivität des Patienten ist, der eben stets denjenigen Phänomenen Bedeutung erteilt (und sie verarbeitet), die er gerade braucht - seien sie nun materieller oder immaterieller Art. Aus semiotischer Sicht kann niemals ein toter Stoff, etwa ein Mineral, einem Lebewesen eine Bedeutung erteilen und diese verarbeiten, sondern immer nur umgekehrt, sodass sich sagen lässt: Nicht das Arzneimittel wirkt, sondern der Patient! Deshalb ist dieser in jedem Fall als real anzuerkennen.

Um diesen letzten Punkt so klar wie möglich zu machen, ist darauf hinzuweisen, dass die Redeweise von arzneilichen «Potenzen», pathogenen «Agenzien» oder krank machenden «Einflüssen» höchst irreführend ist. Sie beruht auf der konventionellen materialistischen Annahme (von der sich auch Hahnemann nicht völlig frei- machen konnte), dass äussere Entitäten eine kausale Wirkung auf den Patienten ausüben. Mit Ausnahme von überwältigenden physikalischen oder chemischen Einwirkungen, wie bei mechanischer Gewalt oder hochdosierten Suppressiva, würden jedoch ohne das Subjekt des Patienten bei regulativen Therapien Arzneimittel überhaupt nicht in Funktionskreise involviert werden, die aber doch in jedem Moment den gegenwärtigen Zustand des Patienten konstituieren. Genauer gesagt, ist nicht der Keim der Protagonist, sondern das individuelle Subjekt ist der Akteur, der krank wird, wenn man ihn bestimmten Dingen aussetzt, die für ihn eine krank machende Bedeutung haben. Und nicht das Arzneimittel verursacht die Heilung, sondern das individuelle Subjekt hat die Fähigkeit (Potenz), auf seine bestimmte Wahrnehmung der Bedeutung von Arzneimitteln zu reagieren, etwa in Form von Verschlimmerung, Verbesserung oder Heilung.

Hahnemann benutzte zwar diese konventionellen Begriffe, die seinen semiotisch-phänomenologischen Ansatz eher verdunkeln als erhellen. Andererseits betonte er aber unermüdlich die sogenannte «dynamische» oder «geistartige» Wechselwirkung zwischen Patient, krankmachendem Einfluss und Arzneimittel. Wenn auch sein Konzept von «dynamisch» noch vage und unscharf war, so war für ihn doch völlig klar, dass homöopathische Heilungen die Kategorien konventionellen physikalischen, chemischen, mechanischen, materiellen und atomistischen Denkens überschreiten [24]. Umso mehr sollten heutige Homöopathen nicht hinter Hahnemann zurückfallen und Ressourcen verschwenden, um gegen konventionelle kausal-mechanische Einwendungen und Bedenken zu argumentieren. Trotz ihrer Komplexität wird die Dynamik des Zeichen-Prozesses, der den Arzneimittelprüfungen und der Heilkunst zugrunde liegt, im semiotischen Modell umfassend repräsentiert.

Um ein Beispiel aus der Lebenswelt zu geben: Wenn es einem Individuum nach der Betrachtung eines bestimmten Bildes an der Wand (oder auf dem Bildschirm) übel wird, dann handelt es sich hier offensichtlich nicht um eine kausale Wirkung des Bildes, die in Doppelblindstudien oder Ähnlichem reproduziert werden könnte. Was hier den Ausschlag gibt, ist vielmehr die individuelle Sensibilität, Disposition, Geschichte usw. des Patienten und seine besondere Dynamik der Bedeutungserteilung und Bedeutungsverarbeitung von Phänomenen, denen er begegnet. Interessanterweise sprach Hahnemann explizit von «Geistes- und Gemüts-Organen» [25], was darauf hinweist, dass er bewusst dieselben Begriffe und Konzepte benutzte, um dynamische Wirkungen auf der physischen und psychischen Ebene gleichermassen zu erklären. Für die konventionelle Medizintheorie ist eine solche Vermischung von Begriffen und Ebenen ein Ärgernis. Im semiotischen Modell wird dagegen prinzipiell verstanden, dass die 
dynamischen Prozesse des Subjekts auf allen Ebenen dieselben sind. Daher ist der Gebrauch von Analogien durchaus gerechtfertigt.

Für Hahnemann war das Ziel der homöopathischen Behandlung, die Gesundheit des Patienten wiederherzustellen, genauer gesagt, ihn in den ursprünglichen Zustand zurückzuversetzen, in dem er sich in seinen gesunden Tagen befand. Wichtig ist hier, dass es Hahnemann nicht darum ging, zu bewerten, ob der genesene Patient mild oder ruppig, gut oder böse, verschämt oder schamlos usw. wurde [26]. Im Gegensatz zur konventionellen akademischen Wissenschaft, deren traditionelle Aufgabe immer darin bestand, allgemeingültige Kriterien und Regeln zu etablieren, um sie dann allem und allen aufzuerlegen, anerkannte und würdigte Hahnemann die unhintergehbare Individualität der Identität des Patienten, was ihn unvermeidlich zu einem Vorläufer des semiotischen Modells macht. Seiner Zeit voraus, schlug Hahnemann nicht abstrakte normative Ideen von Gesundheit oder Ähnliches vor [27], sondern war sich bewusst, dass jedes Subjekt einen individuellen Kern bzw. eine individuelle Stimmung hat, die durch therapeutische Eingriffe nicht verändert werden kann.

Das semiotische Modell erklärt, warum und wie jedes Individuum - zusammen mit seiner subjektiven Umwelt - aus einer frühkindlichen Grundstimmung heraus entspringt, die wiederum die Person in jedem Zustand, den sie erleben wird, begleitet. Wie auch immer sich das Neugeborene gefühlt haben mag, verängstigt, entspannt, neugierig, gierig usw., solange der Erwachsene es schafft, seine ursprüngliche subjektive Umwelt kohärent zu halten, ist er gesund. Jedes Subjekt wird stets versuchen, seinen ersten eingeprägten Zustand mit den Situationen und Phänomenen, mit denen es in seinem späteren Leben interagieren wird, zu harmonisieren bzw. «abzustimmen». Unfähigkeit, die eigene Stimmung und die eigene subjektive Umwelt miteinander zu koordinieren, bedeutet demnach Krankheit. Übersetzt man Hahnemanns Begriff «Lebenskraft» in die Dynamik von Funktions- und Situationskreisen, die das Subjekt mit seiner Umwelt verknüpfen, so findet man sein grundlegendes Konzept in moderner wissenschaftlicher Sprache ausgedrückt.

Schliesslich bleiben auch andere wichtige Positionen Hahnemanns, wie seine teleologischen, deistischen und semiotischen Überlegungen, unverständlich, solange sie aus der Sicht des konventionellen wissenschaftlichen Denkens beurteilt werden. Aus der Perspektive des semiotischen Modells ergibt es jedoch Sinn, wenn Hahnemann behauptet, dass die einzig wissenschaftliche Herangehensweise an den Zustand des Patienten über die Wahrnehmung seiner Zeichen und Symptome durch den Arzt geht. Auch Hahnemanns genaue praktische Anweisungen bezüglich Arzneimittelprüfungen, Fallaufnahme und Wei- terverfolgung des Verlaufs können schwerlich vor dem Hintergrund der konventionellen Medizin angemessen gewürdigt werden, die aus intrinsischen Gründen dazu tendiert, solche Aufgaben auf Fragebögen, Checklisten und Computer zu delegieren. Mit dem semiotischen Modell kann dagegen verstanden und gezeigt werden, dass Zeichen-Prozesse omnipräsent sind und dass Ärzte sich ihrer bewusst sein sollten - in ihrer empathischen Beziehung zu den Patienten wie auch beim Beobachten der Wechselwirkungen zwischen Kranken und den Arzneimitteln, die sie gerade brauchen.

\section{Aufgaben und Ausblick}

Um diese fragmentarische Liste von Beispielen, wie Uexkülls semiotisches Modell des Menschen die lange vernachlässigte Aufgabe der Homöopathie, eine neue wissenschaftliche Theorie zu entwickeln, beflügeln könnte, abzuschliessen, sei daran erinnert, dass Theorie nicht einfach Theorie und Praxis nicht einfach Praxis ist. Ohne stets auch das jeweilige andere zu berücksichtigen und sich der fundamentalen Einheit und dynamischen Verbindung aller Dualitäten bewusst zu sein, wie es das biopsychosoziale Modell nahelegt, könnte nie auf wissenschaftliche Weise das grundlegende Zusammenspiel zwischen Theorie und Praxis, Subjekt und Umwelt oder Patient und Arzneimittel verstanden werden.

Sicherlich hat Hahnemann dies in seinem Innersten verstanden. Die praktischen Anweisungen, die er für die homöopathische Heilkunst fand und etablierte, aus einer Mischung von Intuition, Nachdenken und strukturierter Erfahrung, haben sich jedenfalls über 200 Jahre bewährt.

Dennoch mangelt es der Theorie der Homöopathie noch immer an Konsistenz («Stimmigkeit») und Schlüssigkeit. Sie besteht aus einem breiten Spektrum unterschiedlicher Konzepte und enthält Komponenten sich widersprechender Paradigmen [28, 29]. Die Homöopathie braucht daher eine Revision ihres theoretischen Handwerkszeugs, um den Erfordernissen der heutigen Zeit gerecht zu werden. Sie sollte in der Lage sein, sich als legitime Wissenschaft zu präsentieren, sich mit Nachbarwissenschaften zu vernetzen und mit ihnen auf Augenhöhe zu kommunizieren.

Wie die Geschichte der Medizin zeigt, sind theoretische Konsistenz, Plausibilität und Überzeugungskraft die Basis für soziale, politische und institutionelle Anerkennung $[30,31]$. Wie zu zeigen versucht wurde, wären diese Ziele eventuell erreichbar, würde man das von Thure von Uexküll semiotisch weiterentwickelte biopsychosoziale Modell des Menschen als soliden konzeptuellen Rahmen für eine moderne Theorie der Homöopathie zugrunde legen. 


\section{Disclosure Statement}

Keine Interessenkonflikte.

\section{Literatur}

1 Schmidt JM: Medizintheoretische und wissenschaftshistorische Perspektiven einer Revision der Materia medica homoeopathica. Schweiz Z Ganzheitsmed 2010;22:232-238.

12 Wischner M: Homöopathie und Medizintheorie. Z Klass Hom 2011;55:172-182.

$\checkmark 3$ Schmidt JM: Hahnemanns Homöopathie zwischen rationaler Heilkunde und Heilkunst. Schweiz Z Ganzheitsmed 2011;23:224-232.

4 Schmidt JM: Die esoterische und exoterische Sichtweise der Homöopathie - zwei Seiten derselben Medaille?; in Proceedings 52nd Congress of the Liga Medicorum Homoeopathica Internationalis, May 28June 1, 1997, Seattle/Washington. Edmonds/ Washington, American Institute of Homeopathy, 1997, pp 193-198.

5 Achilles P, Janz D, Schrenk M, von Weizsäcker CF (Hrsg): Viktor von Weizsäcker: Gesammelte Schriften in zehn Bänden. Frankfurt/Main, Suhrkamp, 1986-2005.

6 Bastian T, Hansch D: Thure von Uexküll und die psychosomatische Medizin. Universitas 2004;59:1276-1280.

7 Becker C (Hrsg): Julien Offray de la Mettrie: L'homme machine [1748]. Die Maschine Mensch. Hamburg, Meiner, 2009.

8 NN: The end of homeopathy. Lancet 2005; 366:690.

9 Macke-Bruck B: Thure von Uexküll; in Stumm G, Pritz A, Gumhalter P, Nemeskeri N, Voracek M (Hrsg): Personenlexikon der Psychotherapie. Wien, Springer, 2005, Teil 19, pp 479-481.

10 Engel GL: The need for a new medical model: a challenge for biomedicine. Science 1977;196:129-136.

11 von Uexküll T: Grundfragen der psychosomatischen Medizin. Reinbek, Rowohlt, 1963.
12 von Uexküll T: Die Umweltforschung als subjekt- und objektumgreifende Naturforschung; in von Uexküll J, Kriszat G (Hrsg): Streifzüge durch die Umwelten von Tieren und Menschen. Ein Bilderbuch unsichtbarer Welten. Bedeutungslehre. Frankfurt/Main, Fischer, 1970.

13 Mildenberger F: Umwelt als Vision. Leben und Werk Jakob von Uexkülls (1864-1944). Stuttgart, Franz Steiner, 2007.

14 Krampen M: Thure von Uexküll - Arzt, Wissenschaftler, Semiotiker. Z Semiotik 2004; 26:421-428.

15 Helbach C: Die Umweltlehre Jakob v. Uexkülls: Ein Beispiel für die Genese von Theorien in der Biologie zu Beginn des 20. Jahrhunderts. Phil. Dissertation. Universität Aachen, 1989.

16 Pobojewska A: Die Subjektlehre Jacob v. Uexkülls. Sudhoffs Archiv 1993;77:54-71.

17 Dau T: Die Biologie von Jakob v. Uexküll. Biol Zentralbl 1994;113:219-226.

18 von Uexküll J, Kriszat G: Streifzüge durch die Umwelten von Tieren und Menschen. Berlin, Springer, 1934.

19 Egger JW: Grundlagen der «Psychosomatik». Zur Anwendung des biopsychosozialen Krankheitsmodells in der Praxis. Psychol Med 2008;19:12-22.

20 Storp C: Zur Entstehung der individuellen Wirklichkeit und ihrer Bedeutung in der Medizin im Werk von Thure von Uexküll und Wolfgang Wesiack. Medizinische Dissertation. Universität München, 2009.

21 von Uexküll T, Wesiak W: Theorie der Humanmedizin. Grundlagen ärztlichen Denkens und Handelns, ed 2. München, Urban \& Schwarzenberg, 1991.

22 Schmidt JM (Hrsg): Hahnemann S: Organon der Heilkunst. Neufassung der 6. Auflage mit Systematik und Glossar. München, Elsevier, 2003; ed 2 München, Elsevier, 2006, $\$ \$ 11,29$.
23 Egger JW: Das biopsychosoziale Krankheitsmodell. Grundzüge eines wissenschaftlich begründeten ganzheitlichen Verständnisses von Krankheit. Psychol Med 2005;16:3-12.

24 Schmidt JM: Homöopathie und Philosophie. Versuch einer philosophischen Rekonstruktion der Begründung der Homöopathie durch Samuel Hahnemann. Scheidewege 1990/1991;20:141-165.

25 Schmidt JM (Hrsg): Hahnemann S: Organon der Heilkunst. Textkritische Ausgabe der 6. Auflage. Heidelberg, Haug, 1992; Neuausgabe: Heidelberg, Haug, 1999, $\$ \$ 215-216$.

26 Schmidt JM (Hrsg): Hahnemann S: Organon der Heilkunst. Standardausgabe der 6. Auflage. Heidelberg, Haug, 1996; Neuausgabe: Heidelberg, Haug, 1999, $\$ 210$.

27 Schmidt JM: Gesundheit! - Geschichte und Konzepte des Leitbegriffs der Medizin. Wien Klin Wochenschr 2010;122:538-542.

28 Schmidt JM: Die philosophischen Vorstellungen Samuel Hahnemanns bei der Begründung der Homöopathie (bis zum Organon der rationellen Heilkunde, 1810). München, Sonntag, 1990.

29 Wischner M: Fortschritt oder Sackgasse? Die Konzeption der Homöopathie in Samuel Hahnemanns Spätwerk (1824-1842). Essen, KVC, 2000.

30 Unschuld PU: Was ist Medizin? Westliche und östliche Wege der Heilkunst. München, Beck, 2003.

31 Schmidt JM: Die Entstehung, Verbreitung und Entwicklung von Heilsystemen als $\mathrm{Ge}$ genstand der Medizingeschichte - am Beispiel der Homöopathie. Sudhoffs Archiv 2007; 91:38-72. 